\title{
Magnetic nanowire based high resolution magnetic force microscope probes
}

\author{
G. Yang \\ Department of Physics and Astronomy, University of North Carolina, Chapel Hill, North Carolina 27599 \\ J. Tang and S. Kato \\ National Institute for Materials Science, Tsukuba 305-0047, Japan
}

Q. Zhang and L. C. Qin

Department of Physics and Astronomy and Curriculum in Applied and Materials Sciences, University of North Carolina, Chapel Hill, North Carolina 27599

M. Woodson and J. Liu

Department of Chemistry, Duke University, Durham, North Carolina 27708

J. W. Kim

Science and Technology Corporation, Hampton, Virginia 23666

P. T. Littlehei

Advanced Materials and Processing Branch, MS-226 NASA Langley Research Center, Hampton, Virginia 23681

C. Park

National Institute of Aerospace, MS-226 NASA Langley Research Center, Hampton, Virginia 23681

O. Zhou ${ }^{\text {a) }}$

Department of Physics and Astronomy and Curriculum in Applied and Materials Sciences, University of North Carolina, Chapel Hill, North Carolina 27599

(Received 25 April 2005; accepted 21 July 2005; published online 15 September 2005)

\begin{abstract}
We report an efficient process for controlled fabrication of high-resolution magnetic force microscope probes using preformed magnetic nanowires. Nickel and cobalt nanowires produced by electrodeposition were directly assembled onto the tip of a commercial atomic force microscope cantilever with controlled orientation and length by dielectrophoresis. The properties of these nanowire-based probes are characterized. (C) 2005 American Institute of Physics.
\end{abstract}

[DOI: $10.1063 / 1.2043237$ ]

Magnetic force microscopy (MFM) is a nondestructive experimental technique for investigation of surface magnetic structure of systems such as magnetic storage media. ${ }^{1}$ The resolution and sensitivity of MFM depend largely on the geometry and magnetic properties of the probe. A conventional MFM probe is fabricated by coating the tip of an atomic force microscope (AFM) cantilever with a layer of hard ferromagnetic materials such as a cobalt based alloy. This method increases the tip radius and limits the spatial resolution of the MFM to the order of $100 \mathrm{~nm} .^{1}$ To fabricate MFM probes with reduced radii, several techniques have been investigated which require the use of either electron beam deposition and/or focused ion beam milling. Attachment/ growth of carbon nanotubes (CNTs) with nanometer-size diameters and large aspect ratios on the apex of a Si cantilever has been shown significantly increase the spatial resolution and the probing depth of an AFM. ${ }^{2,3}$ CNT AFM probes are also found to have better wear resistance compared to the $\mathrm{Si}$ probes. ${ }^{4}$ Recently the feasibility of using modified CNTs for high resolution MFM imaging has been reported by several groups. ${ }^{5}$

Three different methods have been developed to fabricate the CNT based MFM probes. Arie et al. mounted a single multiwall carbon nanotube (MWNT) capped with a

\footnotetext{
${ }^{a)}$ Electronic mail: zhou@ physics.unc.edu
}

magnetic catalyst particle onto the apex of a commercial $\mathrm{Si}$ cantilever inside the chamber of a scanning electron microscope (SEM) and reported the first MFM image obtained from a CNT based probe. ${ }^{5}$ Cui et al. demonstrated direct chemical vapor deposition (CVD) growth of a carbon nanofiber on a tipless $\mathrm{Si}$ cantilever. In this tip-growth CVD process, ${ }^{6}$ the encapsulated magnetic particle remains at the top of the nanofiber and provides the magnetic force. ${ }^{6}$ In two recent publications, Kuramochi et $a l^{7}$ and Deng et al. ${ }^{8}$ produced MFM probes by sputtering a layer of magnetic film onto the outer surface of a CNT either mounted ${ }^{7}$ or catalytically grown ${ }^{8}$ on a Si cantilever. The diameter of the resulting magnetic tip is about $40 \mathrm{~nm}^{7}$. Lateral resolution up to 10-20 $\mathrm{nm}$ was demonstrated. Although the imaging results are very promising, the processes of manufacturing these nanoscale CNT magnetic probes still have rooms for improvement. Mechanically mounting a CNT on a Si cantilever inside a SEM, ${ }^{5,7}$ although affords good precision, is difficult to scale up. Direct catalytic growth of CNTs can in principle lead to wafer-scale production, consistency in the tube length and orientation from probe to probe has yet to be demonstrated. Coating magnetic films on the CNT tubes adds additional complexity in the fabrication process.

Magnetic nanowires can now be synthesized by techniques such as electrodeposition with reasonable purity and control of the dimension. Some of these intrinsically magnetic nanowires have diameters and lengths comparable to 


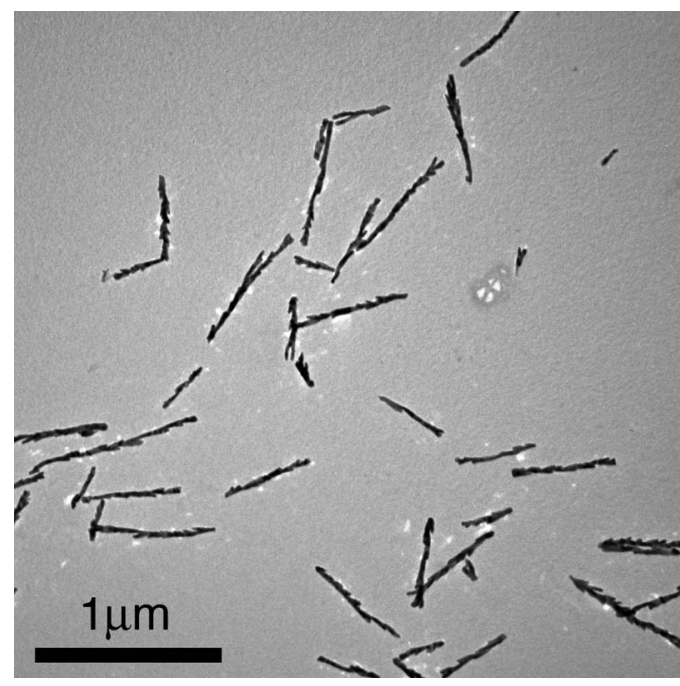

FIG. 1. TEM image of the Ni nanowires used in this study. The nanowires were synthesized by electrodeposition using anodized alumina template. The average diameter is about $40 \mathrm{~nm}$. One side the nanowire has a sawtooth structure.

those of the MWNTs which make them attractive candidates for high resolution MFM imaging. In this letter we investigate the feasibility of fabricating MFM probes by direct assembly of preformed magnetic nanowires onto commercial AFM probes. This is accomplished by a dielectrophoresis process that we recently demonstrated to be effective for controlled assembly of CNTs into point probes such as AFM tips and point emitters. ${ }^{9-11}$ The structure, morphology and imaging capability of these nanowire based magnetic probes are investigated.

Nickel nanowires were fabricated by electrodeposition using anodic alumina template with $15-50 \mathrm{~nm}$ diameter holes. ${ }^{12,13}$ Electrodeposition was carried out at room temperature using $50 \mathrm{~Hz}$ ac current and 15-28 V applied voltage. A water solution containing nickel sulfate and boric acid was used as the electrolyte. After deposition, the nanowires were harvested by dissolving the alumina template in phosphoric acid at room temperature. A representative transmission electron microscope (TEM) image of the Ni nanowires that are nominally $500 \mathrm{~nm}$ in length is shown in Fig. 1. The nanowires have a sawtooth morphology on one side. The length varies from 300 to $800 \mathrm{~nm}$ whereas the diameter is $20-40$ $\mathrm{nm}$. Cobalt nanowires of similar dimensions were also synthesized using the same process. The as-synthesized nanowires were then dispersed in deionized water without using any surfactants, centrifuged, and homogenized in an ultrasonic bath before use.

The dielectrophoresis setup is similar to that we reported previously for assembly of CNTs and is not repeated here. ${ }^{9,10}$ A commercial gold coated Si AFM probe (NSC18/Cr-Au from MikroMasch) was used as the working electrode. An ac field of $2 \mathrm{MHz}$ was applied between the Si cantilever and a counter electrode, which was immersed into the nanowire suspension. Under the guidance of an optical microscope, the $\mathrm{Si}$ cantilever was translated to contact the suspension surface and was then gradually withdrawn under the AC field. The nanowires were found to assemble into a sharp tip with one end anchored on the apex of the Si pyramid via the positive dielectrophoresis process which is expected from metallic objects. ${ }^{14}$ The length of the magnetic tip was controlled by objects. ${ }^{14}$ The length of the magnetic tip was controlled by process with different length and morphology.
Downloaded 15 Sep 2005 to 128.155 .21 .222. Redistribution subject to AlP license or copyright, see http://apl.aip.

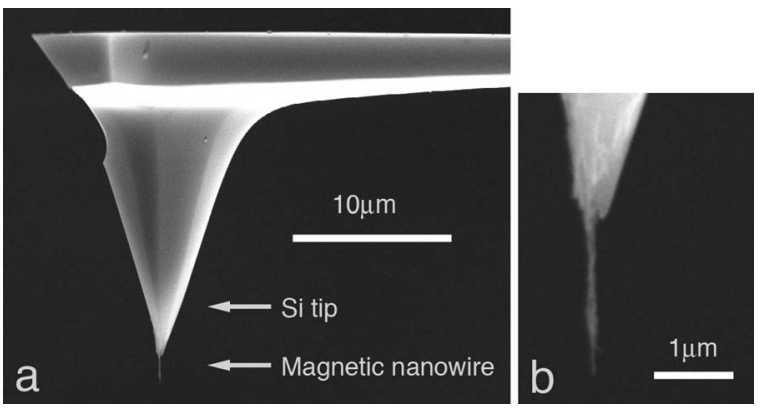

FIG. 2. (a) SEM images of a Ni nanowire bundle attached to a commercial AFM probe by the dielectrophoresis process, (b) high resolution SEM images of the same tip. The nanowire bundle is about $2 \mu \mathrm{m}$ in length and with a single nanowire protrusion at the end.

the distance by which the Si cantilever was translated under the ac field.

SEM images of a probe made using Ni nanowires are shown in Fig. 2. The Ni tip assembled is straight and aligned along the cone axis of the Si cantilever, which is also the direction of the ac electrical field applied during deposition. This particular tip is $\sim 2 \mu \mathrm{m}$ in length and $\sim 40 \mathrm{~nm}$ in diameter at the very tip. It comprises multiple Ni nanowires forming a bundle. One Ni nanowire protruded out from the very end of the bundle giving the small tip diameter. The smallest tip diameter obtained is $\sim 40 \mathrm{~nm}$ which is the same as the diameter of the individual nanowire used for this study. This is comparable to the reported diameters of the MFM probes made from either magnetic particle capped MWNT $(35 \mathrm{~nm})^{5}$ or CNTs wrapped with a layer of magnetic film $(40 \mathrm{~nm}){ }^{7}$ Probes made from the Co nanowires have the same structure and morphology as those by the Ni nanowires. Both are similar to those of the CNT AFM probes made by the dielectrophoresis process. ${ }^{11}$

Over 30 nanowire MFM probes were fabricated by this process and were investigated. The length and the diameter of the tips formed depend on the deposition conditions including the concentration and dispersion of the $\mathrm{Ni} / \mathrm{Co}$ suspension, the ac field strength and the rate by which the $\mathrm{Si}$ cantilever was withdrawn from the suspension surface. Figure 3 shows three Ni MFM probes with different length fabricated by this process. In some of the $\mathrm{Ni} / \mathrm{Co}$ tips, the individual $\mathrm{Ni} / \mathrm{Co}$ nanowires were not closely packed and the overall structure is not straight. One example is shown in Fig. 3(a). These probes were subsequently annealed under $10^{-6}$ Torr vacuum. It was found that the $\mathrm{Ni}$ and $\mathrm{Co}$ nanowires recrystallized into large particles when annealed at temperatures above $800^{\circ} \mathrm{C}$, a temperature substantially lower than their respective bulk-phase melting temperatures. This is attributed to the reduction of the melting temperature

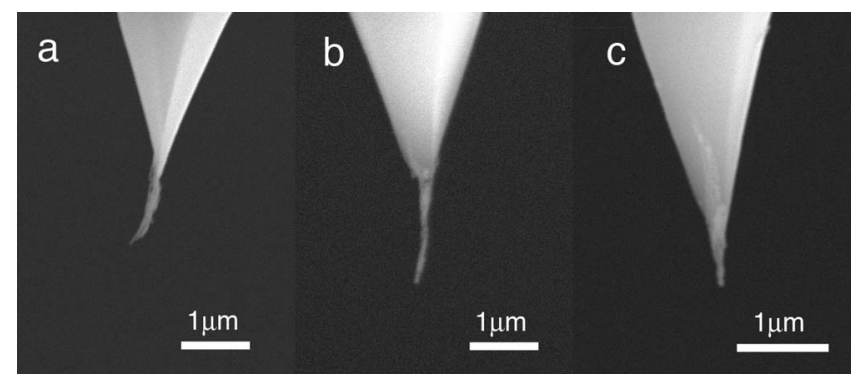

FIG. 3. SEM images of three Ni nanowire MFM probes fabricated by this

process with different length and morphology.
to AIP license or copyright, see http://apl.aip.org/apl/copyright.jsp 

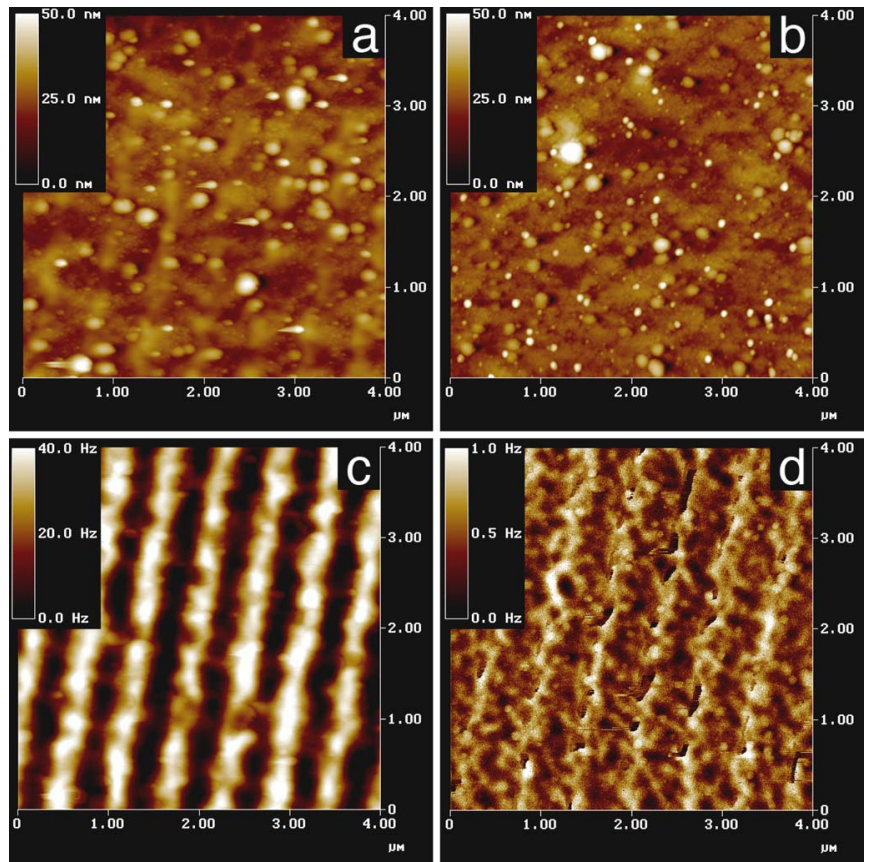

FIG. 4. Topographic and magnetic images of a standard sample measured using a commercial MFM tip (a) and (c) and Ni nanowire based probe (b and d), respectively.

at the nanometer scale. Annealing at $750{ }^{\circ} \mathrm{C}$ for $\sim 1 \mathrm{~h}$ improved the adhesion between the individual nanowires forming the tip, although conglomeration of the metal coating on the Si cantilever was observed after annealing.

Topographic and magnetic images of a standard magnetic recording tape (from Veeco Instrument) were recorded using a laboratory AFM (Digital Instruments NanoScope IIIa). The nanowire probe was magnetized using a permanent magnet prior to imaging. Figure 4 shows two sets of topographic and MFM images obtained from a commercial MFM probe coated with $60 \mathrm{~nm}$ Co and $20 \mathrm{~nm} \mathrm{Cr}$ (NSC18/Co-Cr from MikroMasch) and a Ni probe with $\sim 40 \mathrm{~nm}$ tip diameter [shown in Fig. 3(c)] over a $4 \mu \mathrm{m} \times 4 \mu \mathrm{m}$ area, respectively. The nanowire MFM probe gives a better spatial resolution in topographic imaging than the commercial MFM probe when imaged under the same conditions. Small surface features that were not seen in Fig. 4(a) were clearly resolved in Fig. 4(b). MFM imaging was performed in the frequency mode. As shown in Fig. 4(d), the Ni nanowire tip revealed subtle structures within the magnetic strips of the recording tape that was not resolved by the commercial MFM probe [shown in Fig. 4(c)]. The contrast, however, is lower with the $\mathrm{Ni}$ nanowire probe. The robustness of the nanowire probe was tested by performing 10 scans of the same sample over $4 \times 4 \mu \mathrm{m}$ area. SEM image taken after the measurement shows that tip remained the same.

Figure 5 shows the height profiles of a calibration sample (from Nanoscience) measured using (a) a regular $\mathrm{Si}$ AFM probe (BS-multi75 from Budget Sensor), (b) a magnetic nanowire probe [shown in Fig. 3(a)]; and (c) a CNT AFM probe fabricated by dielectrophoresis. As shown in the figures, the three probes give side angles of 68,78 and 84 degrees, respectively.

The results presented here demonstrate that highresolution MFM probes can be readily fabricated by direct assembly of magnetic nanowires via dielectrophoresis. A tip diameter of $40 \mathrm{~nm}$ was obtained in this study which can be

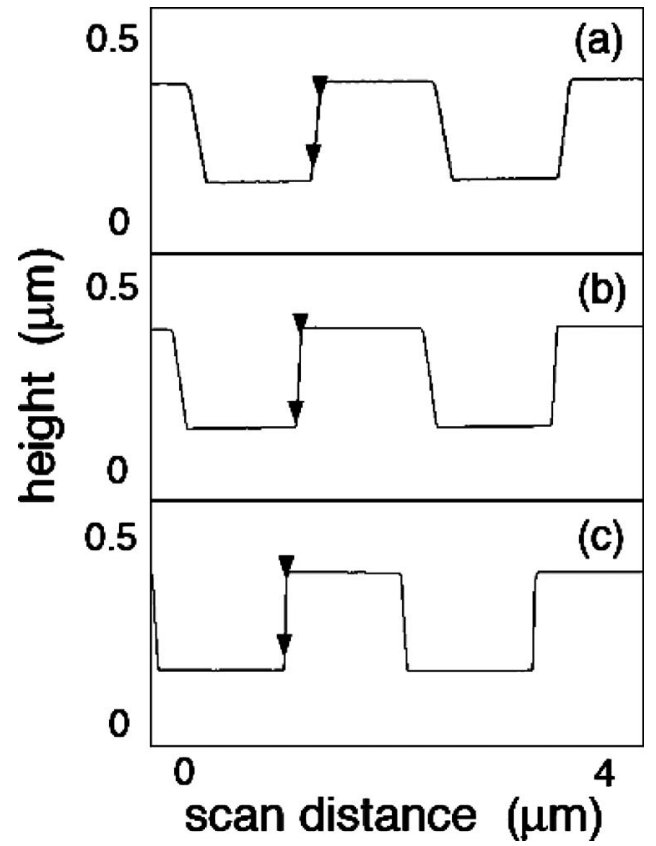

FIG. 5. Height profiles of a calibration sample ( $2 \mu \mathrm{m}$ pitch lines, $200 \mathrm{~nm}$ deep) measured using (a) regular Si AFM probe (BS-multi75 from budget sensor); (b) a Ni nanowire probe; and (c) a CNT AFM probe fabricated by dielectrophoresis. The sidewall angles measured are $68^{\circ}, 78^{\circ}$, and $84^{\circ}$, respectively.

further reduced by using smaller diameter magnetic nanowires. Attachment of magnetic nanowires to regular AFM cantilevers not only provides the capability of magnetic imaging but also extends its probing depth in the topographic mode. The dielectrophoresis process affords reasonable control of the orientation of the magnetic tip.

This work was partially supported by NASA URETI on Bio Inspired Materials (NCC-1-02037), Xintek, Inc., and a Japan-U.S. Cooperative Scientific Program of JSPS. Work done at Duke was supported by AFOSR (49620-02-1-0188). The authors thank J. Hao and R. Superfine for providing some of the magnetic nanowires used early in this study.

${ }^{1}$ U. Hartmann, Annu. Rev. Mater. Sci. 29, 53 (1999).

${ }^{2}$ J. H. Hafner, C.-L. Cheung, A. T. Woolley, and C. M. Lieber, Prog. Biophys. Mol. Biol. 77, 73 (2001).

${ }^{3}$ H. Dai, J. H. Hafner, A. G. Rinzler, D. T. Colbert, and R. E. Smalley, Nature (London) 384, 147 (1996).

${ }^{4}$ T. Larsen, K. Moloni, F. Flack, M. A. Eriksson, M. G. Lagally, and C. T. Black, Appl. Phys. Lett. 80, 1996 (2002).

${ }^{5}$ T. Arie, H. Nishijima, S. Akita, and Y. Nakayama, J. Vac. Sci. Technol. B 18, 104 (2000).

${ }^{6}$ H. Cui, S. V. Kalinin, X. Yang, and D. H. Lowneds, Nano Lett. 4, 2157 (2004).

${ }^{7}$ H. Kuramochi, T. Uzumaki, M. Yasutake, A. Tanaka, H. Akinaga, and H. Yokoyama, Nanotechnology 16, 24 (2005).

${ }^{8}$ Z. Deng, E. Yenilmez, J. Leu, J. E. Hoffman, E. W. J. Straver, H. Dai, and K. A. Moler, Appl. Phys. Lett. 85, 6263 (2004).

${ }^{9}$ J. Tang, B. Gao, H. Geng, O. D. Velev, L. C. Qin, and O. Zhou, Adv. Mater. (Weinheim, Ger.) 15, 1352 (2003).

${ }^{10}$ J. Zhang, J. Tang, G. Yang, Q. Qiu, L. C. Qin, and O. Zhou, Adv. Mater. (Weinheim, Ger.) 16, 1219 (2004).

${ }^{11}$ J. Tang, G. Yang, Q. Zhang, A. Parhat, B. Maynor, J. Liu, L. C. Qin, and O. Zhou, Nano Lett. 5, 11 (2005).

${ }^{12}$ G. J. Strijkers, J. H. J. Dalderop, M. A. A. Broeksteeg, H. J. M. Swagten, and W. J. M. de Jonge, J. Appl. Phys. 86, 5141 (1999).

${ }^{13}$ S. Kato, H. Kitazawa, and G. Kido, J. Magn. Magn. Mater. 272, 1666 (2004).

${ }^{14}$ T. B. Jones, Electromechanics of Particles (Cambridge Universty Press, Cambridge, 1995). 